\title{
Identificação e Controle de um Sistema com um Grau de Liberdade Não Linear Cúbico usando Neutralizadores Dinâmicos Viscoelásticos
}

\author{
Cíntia T. Préve * \\ Universidade Federal do Paraná - UFPR - Pós-Graduação em Engenharia Mecânica \\ 81531-980, Centro Politécnico, Curitiba, PR \\ E-mail: cintiapreve@gmail.com,
}

\author{
Eduardo M. O. Lopes ${ }^{\dagger} \quad$ Carlos A. Bavastri ${ }^{\ddagger}$ \\ Universidade Federal do Paraná - UFPR - Pós-Graduação em Engenharia Mecânica \\ 81531-980, Centro Politécnico, Curitiba, PR \\ E-mail: eduardo_lopes@ufpr.br, bavastri@ufpr.br.
}

\begin{abstract}
RESUMO
Neutralizadores dinâmicos são dispositivos construídos a partir de sistemas ressonantes, utilizados no controle de vibração e ruído irradiado de estruturas mecânicas. Uma metodologia geral de projetos destes dispositivos vem sendo desenvolvida pelo Grupo de Pesquisa de Vibrações e Som em Sistemas Mecânicos (GVIBS) do CNPq nos últimos 20 anos. Através da mesma, um conjunto de neutralizadores dinâmicos viscoelásticos pode ser projetado de forma ótima para reduzir a resposta vibratória em uma ampla faixa de frequência onde uma ou várias frequências naturais estão presentes, com um ou vários dospositivos. Por outro lado, esta metodologia precisa do modelo modal do sistema primário, portanto a premissa básica é que o sistema a controlar seja linear.

A utilização de materias viscoelásticos deve-se a ampla capacidade de dissipação de energia vibratória, bem como a flexibilidade da implementação prática, uma vez que os neutralizadores com amortecimento viscoso são apenas modelos matemáticos a título de comparações.

Neste trabalho, dando continuidade e ampliando esta linha de pesquisa será apresentada uma metodologia para o projeto ótimo de um neutralizador dinâmico viscoelástico atuando em um sistema de um grau de liberdade não linear cúbico. Esta metodologia conta com a identificação do sistema primário e com o projeto ótimo do neutralizador.

A metodologia conta de um problema inverso de identificação através de um ajuste de curvas numérico / experimental por mínimos quadrados com o auxílio de técnicas de otimização não linear; o projeto do neutralizador através de códigos próprios com o uso dos conceitos de parâmetros equivalentes generalizados, modelo a derivada fracionária e técnicas de otimização não linear; realização física do dispositivo de controle e medição de curvas resposta em frequência.

Os resultados obtidos sobre o sistema primário de um grau de liberdade não linear cúbico identificado com e sem neutralizadores dinâmicos viscoelásticos, serão apresentados a fim de validar a eficiência da metodologia.

Palavras-chave: Controle de Vibração, Neutralizadores Dinâmicos Viscoelásticos, Sistemas Não Lineares Cúbicos, Derivada Fracionária, Técnicas de Otimização Não Linear
\end{abstract}

\footnotetext{
*bolsista de Mestrado CAPES

${ }^{\dagger}$ bolsista de Produtividade CNPq

${ }^{\ddagger}$ bolsista de Produtividade CNPq
} 


\section{Referências}

[1] ARORA, Jasbir S. Introduction to Optimum Design. San Diego, California, USA: Elsevier Academic Press, 2004.

[2] BAGLEY, R. L.; TORVIK, P. J. On teh Fractional Calculus Model of Viscoelastic Behavior. Journal of Rheology. 30 (1), p. 133-155, 1986.

[3] BAVASTRI, Carlos A. et al. Optimum Viscoelastic Absorbers for Cubic Non-Linear Systems. Journal of vibration and control. April 2013.

[4] CRUZ, Gilberto A. M. Projeto Ótimo de Neutralizadores Viscoelásticos Baseado no Modelo a Derivadas Fracionárias. Tese de Doutorado em Engenharia Mecânica - UFSC, 2004.

[5] DEN HARTOG, J. P. Mechanical Vibrations. New York: McGraw-Hill, 1956.

[6] DOUBRAWA FILHO, Francisco J. Controle de Vibração Flexional em Sistemas Girantes utilizando Neutralizadores Dinâmicos Viscoelásticos. Dissertação de Mestrado em Engenharia Mecânica - UTFPR, 2008.

[7] ESPÍNDOLA, J. J.; BAVASTRI, C. A.; LOPES, E. M. O. On the Passive Control of Vibrations with Viscoelastic Dynamic Absorbers os Ordinary and Pendulum Types. Journal of the Franklin Isntitute. Vol. 347, 102-115, 2010.

[8] ESPÍNDOLA, J. J.; BAVASTRI, C. A.; CRUZ, G. A. M. On the Design Optimum Systems of Viscoelastic Vibration Neutralizers. In Modelling and Control of Autonomous Decision Support Based Systems, E. P. Hofer e E, Reithmeier, eds., p. 49-64, 2005 a.

[9] ESPÍNDOLA, J. J.; SILVA, H. P. Modal Reduction of Vibrations by Dynamic Neutralizers: A generalized approach. IMAC-10-10 th International Modal Analysis Conference. Society for Experimental Mechanics, San Diego. Vol. 2, p. 1367-1373, 1992.

[10] FINDLEY, W. N.; LAI, J. S.; ONARAN, K. Creep and Relaxation of Nonlinear Viscoelastic Materials with an Introductin to Linear Viscoelasticity. Amsterdam: North-Holland Publishing Company, 1976.

[11] GONÇALVES, Vinícius V. Projeto de Neutralizadores Viscoelásticos para Sistemas Mecânicos Não Lineares. Relatório de Iniciação Científica do Curso de Graduação em engenharia Mecânica - UFPR, 2012.

[12] KITIS, L. Vibration Reduction over a Frequency Range. Journal of Sound and Vibration. Vol. $89, \mathrm{n}^{\circ} 4$, p. 559-569, 1983.

[13] NAYFEH, A.; MOOK, D. T. Nonlinear Oscillations. John Wiley and Sons, 1979.

[14] NELDER, A.; Mead, R. A Simplex Method for Function Minimization. In: Computer Journal. Col. 7, p. 308, 1965.

[15] PRITZ, T. Analysis of four-parameter fractional derivative model of real solid materials. Journal of Sound and Vibration. 195 (1), p. 103-115, 1996.

[16] THOMSEN, Jon J. Vibrations and Stability: Order and Chaos. England: McGraw-Hill, 1997.

[17] WORDEN, K.; TOMLINSON, G. R. Nonlinearity is Structural Dynamics: Detection, Identification and Modelling. Institute of Physics Publishing, Bistrol and Philadelphia, 2001.

[18] ZHOU, Shiyu; SHI, Jianjun. Active Balancing and Vibration Control of Rotating Machinery: A survey. The Shock and Vibration Digest. p. 360-371, September 2001. 